\title{
Tissue distribution and cellular localization of gold nanocarriers with bound oligonucleotides
}

\author{
Nayab Fatima1, Ugur Akcan², Mehmet Kaya², Radka Gromnicova1, Jane Loughlin¹, Basil \\ Sharrack $^{3} \&$ David Male*,1 (iD) \\ ${ }^{1}$ Department of Life, Health \& Chemical Sciences, The Open University, Milton Keynes, UK \\ ${ }^{2}$ Department of Physiology, Koç University School of Medicine, Istanbul, Turkey \\ ${ }^{3}$ Academic Department of Neuroscience \& Sheffield, NIHR Translational Neuroscience BRC, Sheffield Teaching Hospitals, NHS \\ Foundation Trust, University of Sheffield, Sheffield, UK \\ *Author for correspondence: Tel.: +44 190865 9226; David.Male@Open.ac.uk
}

\begin{abstract}
Aim: The aim of the study was to determine how the addition of a DNA oligonucleotide cargo to 3$\mathrm{nm}$ gold glyconanoparticles would affect tissue distribution. Methods: Gold glyconanoparticles with 1-6 covalently bound oligonucleotides (40 nt dsDNA) were injected into rats and allowed to circulate for $10 \mathrm{~min}$. Organs were harvested and gold quantitated by inductively coupled plasma mass spectrometry. Cellular localization of the nanocarriers was determined by electron microscopy. Results $\&$ conclusion: Addition of DNA cargo to the nanocarriers prevented localization in the kidney but increased localization in liver hepatocytes and splenic macrophages. There was no significant change in heart, lung or brain. DNA increases the size and adds a strong negative charge to the nanoparticles, which radically affects tissue distribution.
\end{abstract}

Keywords: brain • DNA oligonucleotides $\bullet$ electron microscopy $\bullet$ gold nanoparticles $\bullet$ kidney $\bullet$ liver $\bullet$ spleen $\bullet$ tissue distribution

In recent years, there has been great interest in the potential for gene therapy or gene editing to treat conditions caused by single-gene defects, many of which affect the CNS [1]. One approach is the use of miRNAs, lncRNAs and siRNA to suppress production of defective proteins. Another technique is direct editing of the defective gene in tissue cells. Both methods require delivery of oligonucleotides to specific cell types in the target tissue. Although direct injection into a site in the target tissue is possible, delivery of the treatment by intravascular infusion is less invasive and may allow a better distribution in the tissue. Treating diseases of the brain is particularly difficult as the tight junctions between brain endothelial cells prevent movement of large biomolecules, including oligonucleotides, across the blood-brain barrier (BBB) [2]. The more general problem is that any treatment delivered systemically usually has to cross vascular endothelium before it can reach cells in the tissue [3]. The ability of large biomolecules to cross the endothelium either by a paracellular or transcellular route depends on the type of microvascular endothelium within each tissue.

Various studies have used nanoparticles (nanocarriers) to deliver an oligonucleotide cargo to cells. There are two basic types of such nanocarrier: nanoparticles with the oligonucleotide incorporated into the core, which are usually $>50 \mathrm{~nm}$ in diameter, or smaller nanocarriers with the oligonucleotides bound to the outside. Earlier studies in vitro had shown that gold nanoparticles (GNPs) with a 1.5-nm core and a coat of covalently bound sugar molecules (total diameter $\sim 3 \mathrm{~nm}$ ) cross human brain endothelial cells [4] and are taken up by kidney endothelial cells and lung microvascular endothelium at considerably higher levels than the brain endothelium [4,5]. Transport occurs partly by vesicular transcytosis and partly by direct movement across the apical and basal plasma membranes via the cytosol, depending on the formulation and charge of the nanoparticle [5].

These gold glyconanoparticles (sugar-coated GNPs) are particularly versatile as carriers of therapeutic biomolecules because the bound sugar lends itself to an 'exchange reaction' with other biomolecules (cargo) that have a free - SH group. This technique has been used to attach oligonucleotides and polypeptides to the nanoparticles. The conjugates are stable for months when stored in an oxidizing environment but progressively

Future $\because$ Medicine 
release their cargo in a reducing environment. To have a biological effect, nanocarriers with bound oligonucleotides must enter the cytoplasm of the target cell and then release their cargo. These 3-nm gold glyconanoparticles are small enough to enter the cytosol by transfer across the plasma membrane [6], and they can then release their cargo by exchange reaction with glutathione in the cytoplasm [7]. The potential for disease treatment has been demonstrated by studies showing that gold-RNA or gold-DNA conjugates can modulate gene expression [8], confirming that the transported oligonucleotides retain their biological activity after internalization by target cells. Another advantage of GNPs is their safety [9]. Gold glyconanoparticles similar to those used in this study but with different cargoes have been US FDA-approved for treatment of glioma [10] and are already used in clinical trials for treatment of diabetes.

In vivo, the bound coat of sugar molecules maintains solubility, prevents aggregation and limits removal of nanoparticles by the mononuclear phagocyte system. However, one drawback of smaller nanoparticles $(<5 \mathrm{~nm})$ is that they tend to become localized in the kidney and/or excreted in urine. It has been assumed that their small size allows them to pass through the fenestrated endothelium of the glomerulus [11], and there is no mechanism for reabsorption. Examination of the tissue distribution of glucose-coated GNPs by inductively coupled plasma (ICP) mass spectrometry, $10 \mathrm{~min}$ after intravascular infusion into rats, showed that the majority were localized in the kidney, with much smaller amounts in cerebral cortex, lung and liver [12]. To increase the half-life and availability of these very small nanocarriers, it would be necessary to reduce losses through the kidney.

Attachment of cargo molecules to the glyconanoparticles can radically alter their physicochemical characteristics and their interaction with endothelial cells. For example, coupling 40-nt double-stranded DNA oligonucleotides to the outside of the nanoparticles makes them negatively charged, increases their hydrodynamic diameter to approximately $7 \mathrm{~nm}$ and increases the rate of transcytosis of the carriers up to tenfold and cargo 50-fold [13].

The aim of this study was to determine whether attachment of an oligonucleotide cargo to the gold nanocarriers affects their tissue distribution and cellular localization in vivo. Two formulations of nanoparticle were used, one with 1-3 bound oligonucleotides per nanoparticle (NP-DNA-40 ${ }^{\mathrm{LO}}$ ) and the other with 3-6 oligonucleotides per nanoparticle (NP-DNA-40 ${ }^{\mathrm{HI}}$ ) [13]. They were compared with the unconjugated glyconanoparticle (NP-Gal). Double-stranded DNA of 40-nt length was chosen as the cargo molecule because it is less susceptible to degradation than RNA, and this length of oligonucleotide is of the size typically used for therapeutic purposes.

The results demonstrate that addition of the oligonucleotide cargo causes a major tissue redistribution of the nanoparticles in vivo, markedly reducing excretion by the kidney and increasing uptake by hepatocytes. It shows that changes to the size, charge and formulation of a nanocarrier of this type will have a substantial effect on its bioavailability and the potential for targeting the nanocarrier to a specific tissue. These nanocarriers would be very effective for delivering oligonucleotides to liver hepatocytes, but delivery to other tissues would require additional targeting molecules on the nanoparticles.

\section{Materials \& methods}

Gold glyconanoparticles, oligonucleotides \& attachment to nanoparticles

The GNPs, obtained from Midatech Pharma Plc (Cardiff, UK), were synthesized using a modified Brust-Schiffrin method [14] and characterized in water using dynamic light scattering (DLS) on a Nano ZSP Zetasizer (Malvern Instruments, Malvern, UK). They were capped during synthesis with thiol-C2-galactose. The characteristics of these glyconanoparticles (NP-Gal) are summarized in Supplementary Figure 1.

The method for production and characterization of the gold glyconanoparticles with bound oligonucleotides has been described previously [13]. It involves a chemical exchange reaction in which the ligand with a free - $\mathrm{SH}$ group exchanges with the $\mathrm{C} 2$-galactose which is bound to the gold core via a sulfur atom. In this case, a 20-nt, thiolated ssDNA oligonucleotide (5' thiol-C6-AAT ATC GCG GAC AGA AGA CG 3'), obtained from SigmaAldrich (Gillingham, UK), was attached to the NP-Gal by a 48-hour exchange reaction. Before attachment to the glyconanoparticles, Tris(2-carboxyethyl)phosphine (TCEP), in $0.1 \mathrm{M}$ tris $/ \mathrm{HCl}, \mathrm{pH} 7.5$, was used to reduce the DNA to yield free thiol monomers. This was achieved by incubating the oligonucleotide $(7.5 \mathrm{mM}$ in $50 \mu \mathrm{l})$ at room temperature for $4 \mathrm{~h}$ with TCEP at a molar ratio of 1: 1.25 (DNA:TCEP). After reduction, the oligonucleotide was attached to the NPs by an exchange reaction. NPs $(2 \mathrm{mg} / \mathrm{ml}$ equivalent of gold $)$ were mixed with reduced $20 \mathrm{nt}$ ssDNA at a molar ratio of 1:14 in a $0.5 \mathrm{ml}$ Eppendorf tube, in a sealed container flushed with nitrogen gas to exclude oxygen. The reaction was allowed to continue for $48 \mathrm{~h}$ at room temperature.

To synthesize nanoparticles with 40-nt double-stranded DNA, this NP-DNA-2 $0^{\text {ss }}$ preparation was first hybridized with an equimolar amount of $40 \mathrm{nt} s \mathrm{DNA}$, with its $3^{\prime}$ sequence complementary to the 20-nt ssDNA bound to the NP (5'-AAAAGCTCTGCCTTGGTTTCCGTCTTCTGTCCGCGATATT-3') for $30 \mathrm{~min}$. Then, 
Figure 1. Five groups of rats (three per group) were treated with the different nanoparticle formulations (top) according to the schedule shown in the figure.

NP: Nanoparticle; NP-Gal: Glyconanoparticle.

\begin{tabular}{|c|c|c|}
\hline Formulation & Intra-carotid & Intravenous \\
\hline NP-gal & 3 & 3 \\
\hline NP-DNA-40 40 & 3 & - \\
\hline NP-DNA-40 ${ }^{\mathrm{HI}}$ & 3 & 3 \\
\hline
\end{tabular}

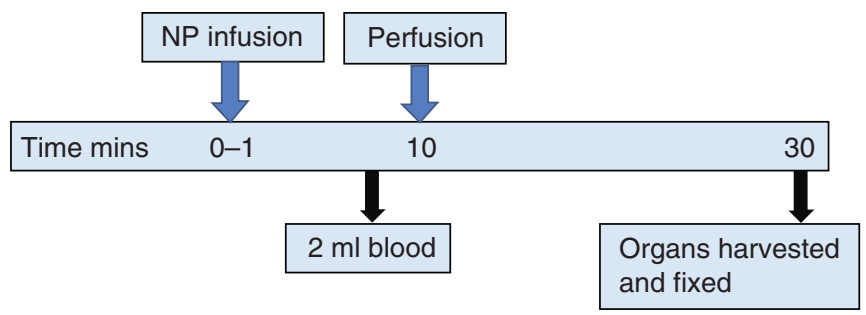

the extended single-strand of the 40-nt oligonucleotide was filled in by hybridization with an equimolar amount of 20-nt ssDNA (5'-GAAACCAAGGCAGAGCTTTT-3'). A diagram showing the arrangement of the different oligonucleotides attached to the gold core is shown in Supplementary Figure 2.

The reaction products were then fractionated on an ÄKTA pure FPLC system (GE Healthcare, Amersham, UK) using a Superdex 200 10/300 GL column in phosphate-buffered saline (PBS). This procedure removes any unbound oligonucleotides and unconjugated NP-Gal. It also provides data on the hydrodynamic diameter of the NPs and fractionates the conjugated NP-Gal-oligonucleotides into separate pools with high or low levels of bound oligonucleotides designated NP-DNA- $40^{\mathrm{HI}}$ and NP-DNA- $40^{\mathrm{LO}}$.

Gold in the nanoparticle formulations was measured by a spectrophotometric method against gold standards (Sigma-Aldrich). The assay was performed in a 96-well plate, with final volumes of $200 \mu$ l. In each well, $10 \mu \mathrm{l}$ of sample, $10 \mu \mathrm{l} \mathrm{H}_{2} \mathrm{O}$ and $30 \mu \mathrm{l}$ of $100 \%$ freshly prepared aqua regia (kept on ice) were added. The liquids were then mixed by gentle tapping and left for $1 \mathrm{~min}$ to dissolve the gold core of the nanoparticles. Next, $150 \mu \mathrm{l}$ of $2 \mathrm{M}$ $\mathrm{NaBr}$ was added. The absorbance was read on an OPTIMA FluoSTAR plate reader, at $390 \mathrm{~nm}$. The concentrations $(\mu \mathrm{g} / \mathrm{ml})$ of NPs used in the biological assays refer to the gold concentration.

\section{Intravascular infusion of NP-Gal with bound oligonucleotides}

All experimental procedures were reviewed and approved by the Local Ethics Committee for Animal Experimentation of Koç University, Istanbul, Turkey (2018.HADYEK.010), and were conducted in adherence to the NIH Guide for the Care and Use of Laboratory Animals. The animals used in this study were purchased from the Experimental Animal Laboratory of Koç University Research Centre for Translational Medicine. Experimental animals were housed in a climate-controlled room (temperature: $22 \pm 1{ }^{\circ} \mathrm{C}$; humidity: $55 \pm 5 \%$ ) and supplied with drinking water and food ad libitum. Five groups of Wistar Albino male rats (age approximately 3 months, weighing $220-340 \mathrm{~g}$ ), three rats per group were used in the study (Figure 1). The experimental protocols were chosen to match and allow comparison with a previous study using gold glyconanoparticles [12]. Intracarotid infusion was chosen as a potential method for optimizing delivery to the CNS, and i.v. infusion requires less technical expertise and is more widely used for intravascular delivery of therapeutic agents.

On the day of the experiment, rats were anesthetized with ketamine $(100 \mathrm{mg} / \mathrm{kg}$, i.p.) $/$ xylazine $(10 \mathrm{mg} / \mathrm{kg}$, i.p.). For intracarotid infusion, the left common internal and external carotid arteries were exposed. The latter was then retrogradely catheterized with a polyethylene tube (PE-10) after its branches were ligated. The tip of the catheter was placed and implanted in the external carotid artery approximately $1 \mathrm{~mm}$ distal to the carotid bifurcation to infuse nanoparticles. Each rat was injected with $200 \mu \mathrm{g} / \mathrm{kg}$ rat body weight of NP-Gal, NP-DNA-40 ${ }^{\mathrm{LO}}$ or NP-DNA- $40^{\mathrm{HI}}$ (gold concentration) over $1 \mathrm{~min}$ into the left internal carotid artery (for i.c. injection) and the penile vein (for i.v. injection). The nanoparticles were left to circulate for $10 \mathrm{~min}$ in the blood (Figure 1). This time was selected as it allows a first pass through the brain before passage through the pulmonary circulation and distribution in the systemic circulation, before any significant level of excretion can occur. After $10 \mathrm{~min}$, the thoracic cavity was exposed following an additional i.p. administration of ketamine $(50 \mathrm{mg} / \mathrm{kg}$, i.p.) with xylazine $(5 \mathrm{mg} / \mathrm{kg})$. Two milliliters of blood were collected from the heart and stored in heparin, and the animals were transcardially perfused for approximately $20 \mathrm{~min}$ with $75 \mathrm{ml}$ of $0.9 \%$ saline to remove the remaining blood. Post perfusion, brain, kidney, liver, lung, spleen and heart were collected. For paired organs, one was fixed and 
the other frozen. The other organs were cut in half, and one half was fixed in $2 \%$ paraformaldehyde and $2.5 \%$ glutaraldehyde and the other half was frozen. (Brain was split coronally at bregma +1 and the anterior portion fixed and the posterior portion frozen.) Fixed samples were used for electron microscopy and frozen samples for inductively coupled plasma mass spectrometry (ICP-MS). For the ICP-MS, the tissue samples were digested with $25 \%$ tetramethyl ammonium hydroxide with $0.2 \%$ Triton $\times 100$, and neutralized with $1 \% \mathrm{HCl}$. The analysis was carried out at Midatech Pharma plc, Bilbao, and results expressed as nanograms of gold per gram of tissue.

\section{Tissue staining \& transmission electron microscopy}

The tissues for transmission electron microscopy (TEM) analysis were kept in fixative for $2 \mathrm{~h}$ at $4^{\circ} \mathrm{C}$ and then stored in PBS at $4^{\circ} \mathrm{C}$. Tissue sections of $100-\mu \mathrm{m}$ thickness were cut with a microtome and placed in $0.1 \mathrm{M}$ Sørensens phosphate buffer $(\mathrm{PB})$ at $4^{\circ} \mathrm{C}$ or frozen at $-20^{\circ} \mathrm{C}$ in $\mathrm{PB}$ with sucrose and ethylene glycol until use.

The sections were stained for 40 min using Aurion LM silver enhancer according to the manufacturer's instructions and then imaged using a light microscope.

An adjacent tissue section to the one stained with LM silver enhancer was selected, and highly stained regions of interest were cut from that tissue section. Selected sections for all groups for all organs were placed in glass vials and permeabilized with $0.05 \%$ Triton $\times 100$ for 30 min and washed $3 \times$ with $0.1 \mathrm{M}$ PB. Sufficient Aurion EM silver enhancer $(\sim 150 \mu \mathrm{l})$ was applied to cover each tissue sample for $2 \mathrm{~h}$ at room temperature. Tissues were washed $3 \times$ with distilled water, and $1 \%$ osmium tetroxide in $\mathrm{PB}$ was applied for 45 mins at room temperature. The tissue samples were rewashed $3 \times$ with $0.1 \mathrm{M} \mathrm{PB}$, and dehydrated in acetone (30\%, $10 \mathrm{~min}: 50 \%, 10 \mathrm{~min}: 90 \%, 10 \mathrm{~min}$ : $100 \%$ twice, 20 min each: $100 \%$ with molecular sieve, $20 \mathrm{~min}$ ).

After dehydration, the tissue sections were incubated in a 50:50 mixture of 100\% acetone and Epon resin and placed on a rocker overnight. The next day, the tissue sections were penetrated with fresh resin twice for $2 \mathrm{~h}$. After that, tissue sections were flat embedded in Aclar sheets and incubated for $48 \mathrm{~h}$ at $60^{\circ} \mathrm{C}$. Meanwhile, capsule-shaped resin blocks were prepared using gelatin capsules as shape former and 13-15 drops of resin added and incubated for $48 \mathrm{~h}$ at $60^{\circ} \mathrm{C}$. After $48 \mathrm{~h}$, the tissue section embedded in Aclar sheets was glued onto the resin capsules and micro-sectioned for TEM analysis. Ultrathin sections of $80 \mathrm{~nm}$ were cut and counterstained with $3 \%$ uranyl acetate for $30 \mathrm{~min}$ followed by Reynolds lead citrate for $10 \mathrm{~min}$. The sections were imaged on a JEOL 1010 electron microscope at $80 \mathrm{kV}$.

\section{Statistical methods}

Data were analyzed, and graphs prepared using Graphpad Prism 8.0. Tests used, replication and p-values are reported in the figure legends.

\section{Results}

\section{Gold glyconanoparticles \& DNA-conjugated glyconanoparticles}

The gold glyconanoparticles used in this study have a gold core with a surface coat of covalently bound C2galactose. The spherical gold core of the nanoparticles was $1.3 \mathrm{~nm}$ in diameter, as determined by transmission electron microscopy, and the size of the glyconanoparticles was $3.63 \pm 1.09 \mathrm{~nm}$ (mean \pm SD) measured by DLS with a zeta potential of $-28.6 \pm 7.01 \mathrm{mV}$. As determined by molecular mass of the gold core $(\sim 20,000)$ and physical size, these nanoparticles have approximately 100 gold atoms in the core and 40 covalently bound C2-galactose molecules on the outside. The characteristics of these nanoparticles were reported previously [13] and are summarized in Supplementary Figure 1. These glyconanoparticles are designated 'NP-Gal'.

DNA oligonucleotides (40 nt length, Supplementary Figure 2) were attached to the gold core of the NP-Gal by an exchange reaction. The size of the gold core of the DNA-conjugated NP-Gal was determined by TEM (Supplementary Figures $3 \& 4$ ) and the hydrodynamic diameter by FPLC gel filtration. These data have been reported previously [13] and are summarized in Supplementary Figure 1.

Two formulations of DNA-conjugated gold glyconanoparticles were prepared with either low levels of covalently bound oligonucleotides (NP-DNA- $40^{\mathrm{LO}}$ ) or high levels of oligonucleotides (NP-DNA- $40^{\mathrm{HI}}$ ). The two formulations were examined by an electrophoretic mobility shift assay (Supplementary Figure 5) the NP-DNA- $40{ }^{\mathrm{HI}}$ preparation had 3-6 bound oligonucleotides per NP-Gal carrier and the NP-DNA- $40^{\mathrm{LO}}$ preparation had $1-3$ oligonucleotides per NP-Gal [13,15]. 


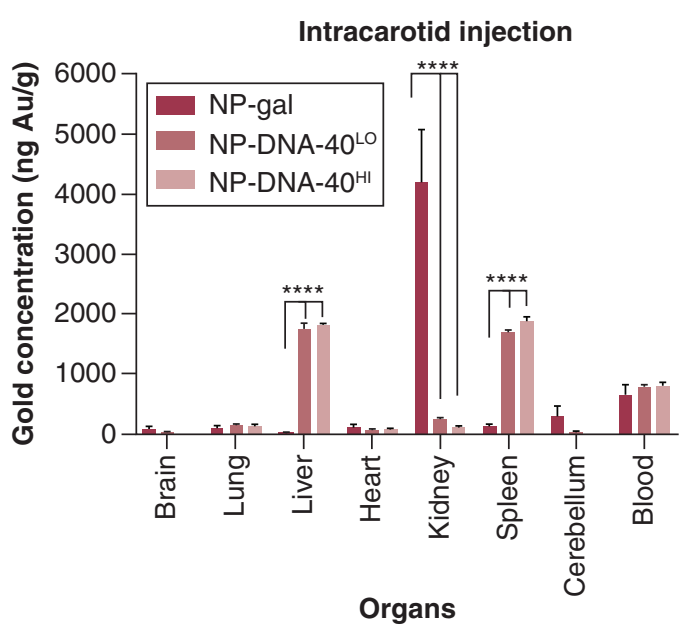

Figure 2. The amount of gold per gram of tissue in different organs analyzed by inductively coupled plasma MS $10 \mathrm{~min}$ after intracarotid infusion (mean \pm standard error of the mean, three animals in each group). Data were analyzed by Dunnett's multiple comparison test. Significant difference was detected between the NP-Gal compared with NP-DNA-40 ${ }^{\mathrm{LO}}$ and NP-DNA- $40^{\mathrm{HI}}$ for kidney, liver and spleen. $* * * * \mathrm{p}<0.0001$. NP: Nanoparticle; NP-Gal: Glyconanoparticle.

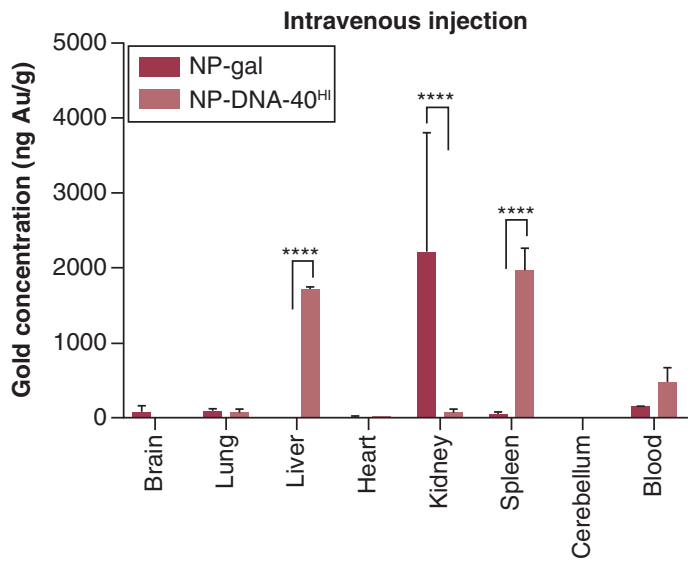

Organs
Figure 3. The amount of gold per gram of tissue in different organs analyzed by inductively coupled plasma MS $10 \mathrm{~min}$ after intravenous infusion (mean \pm standard error of the mean, three animals in each group). Data were analyzed by Dunnett's multiple comparison test. Significant difference was detected between the NP-Gal and NP-DNA- $40^{\mathrm{HI}}$ for kidney, liver and spleen.

$* * * * p<0.0001$.

NP: Nanoparticle; NP-Gal: Glyconanoparticle.

\section{Tissue distribution of gold nanocarriers with bound oligonucleotides}

The nanoparticles were infused into the internal carotid artery of rats over the course of $1 \mathrm{~min}$ and were allowed to circulate for $10 \mathrm{~min}$ before the animals were perfused and tissues collected. The quantity of gold in different tissues was measured by ICP-MS (Figure 2).

It was noted that the unconjugated glyconanoparticles, NP-Gal, accumulated in kidney 10 min after infusion. This finding was as expected for nanoparticles $<5 \mathrm{~nm}[16]$ because they are cleared through the fenestrated endothelium of glomerular capillaries. The results were comparable to a previous study with glucose-coated GNPs of a similar size [12]. Compared with kidney ( $4329 \mathrm{ng} / \mathrm{g}$ ), the concentration of NP-Gal was much smaller in the brain $(91 \mathrm{ng} / \mathrm{g})$, liver $(50 \mathrm{ng} / \mathrm{g})$, spleen $(132 \mathrm{ng} / \mathrm{g})$ and other organs, whereas some remained in the blood $(657 \mathrm{ng} / \mathrm{g})$.

For both, NP-DNA- $40^{\mathrm{LO}}$ and NP-DNA- $40^{\mathrm{HI}}$, the nanoparticles accumulated at high levels in the liver and spleen, and there was almost no accumulation in kidney. The tissue distribution following intravenous infusion (Figure 3) was very similar to the results after intracarotid infusion. Addition of oligonucleotides to the nanoparticles stopped accumulation in the kidney but caused accumulation in liver and spleen, with no significant change in heart, lung or brain. Because addition of oligonucleotides to these carriers increases their rate of transcytosis across brain endothelium in vitro [13], one aim of this experiment was to determine whether addition of oligonucleotides would increase passage across brain endothelium in vivo. However, the rapid accumulation of the NP-DNA-40 nanocarriers in liver and spleen meant that relatively low levels of nanoparticles remained in the blood, and therefore the amount available in the circulation to cross the brain endothelium was also low. 


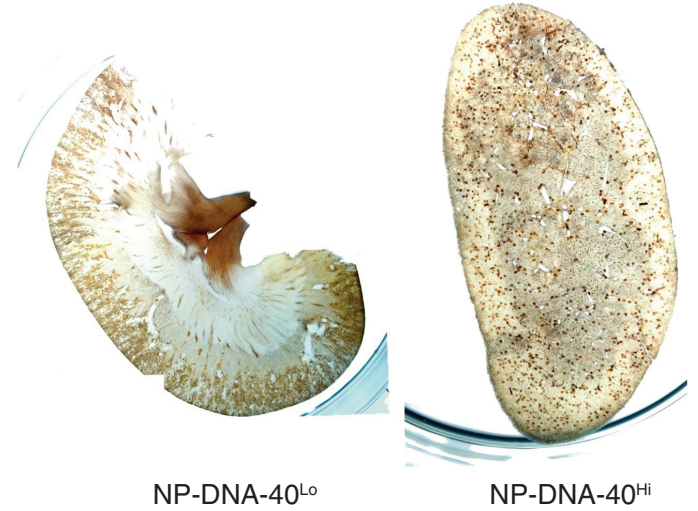

Figure 4. Silver staining of gold nanoparticles in kidney sections of rats following infusion with NP-DNA- $40^{\mathrm{LO}}$ and NP-DNA-40 ${ }^{\mathrm{HI}}$. NP: Nanoparticle.

\section{Cellular distribution in the kidney}

To understand the basis for the change in tissue distribution of nanocarriers caused by attachment of oligonucleotides, the tissues were examined by light and electron microscopy. We hypothesized that the lack of NP accumulation in kidney was due to their inability to cross the fenestrated glomerular endothelium. This could be explained by the increased size and/or high negative charge of the NP-DNA-40 nanocarriers.

Macroscopic examination of GNPs was performed using silver enhancement, which reacts with GNPs and increases their size by depositing silver on their surface. Where the concentration of GNPs (and therefore silver) is high in a tissue, such areas appear brown.

Silver-enhanced kidney sections of animals treated with NP-Gal demonstrated staining throughout the kidney including the renal pelvis. Staining was lower for animals treated with NP-DNA- $40^{\mathrm{HI}}$ and was primarily in the Bowman capsules, appearing as dark spots on the cortex (Figure 4). For animals treated with NP-DNA-40 ${ }^{\mathrm{LO}}$ staining was seen in the Bowman capsules, cortex and in the loops of Henle, with a small amount in the renal pelvis (Figure 4). There was no difference related to whether the NP-DNA-40 nanoparticles had been injected intravenously or into the carotid artery.

When observed by TEM, GNPs are intensely electron-dense and appear as black spots on the tissue, readily distinguishable from cellular structures. NP-Gal nanoparticles in the kidney were located in the Bowman capsule and in the brush borders of the proximal tubular epithelium indicating that they had already passed through the glomerular filtration barrier (Figure 5). In animals treated with NP-DNA- $40^{\mathrm{LO}}$ and NP-DNA- $40^{\mathrm{HI}}$, smaller numbers of nanoparticles were observed in the proximal tubular epithelium and brush border (Supplementary Figure 6).

\section{Cellular distribution in the liver \& spleen}

Although the NP-DNA-40 nanocarriers did not accumulate in the kidney, this alone does not account for the concomitant large increase in liver and spleen; if the increase was due to an increase in bioavailability, one would expect also to see an increase in lung and heart and this did not happen (Figures $2 \& 3$ ). We hypothesized that the increased size or negative charge of NP-DNA- $40^{\mathrm{LO}}$ and NP-DNA- $40^{\mathrm{HI}}$ may have facilitated their accumulation in mononuclear phagocytes and Kupffer cells in the liver - the capillaries in the liver and spleen are discontinuous and lined by phagocytes and are therefore directly accessible to the nanocarriers [17].

In accordance with the ICP-MS data, silver staining of the liver sections was stronger in animals treated with NP-DNA- $40^{\mathrm{LO}}$ or NP-DNA- $40^{\mathrm{HI}}$ compared with NP-Gal. However, this was difficult to visualize in macroscopic sections because of the intrinsic color of the organ.

TEM images showed that in all cases the nanoparticles were primarily localized in the cytoplasm of hepatocytes. Contrary to the original hypothesis, no nanoparticles were observed in Kupffer cells for any of the three groups (Figure 6).

In the spleen, it was not possible to see differences in the level of silver staining macroscopically due to the intrinsic dark color of the organ. In the TEM images, there was a small number of nanoparticles in the red pulp, for animals treated with NP-Gal, but none in the white pulp and the capsule. For animals treated with NP-DNA-40 ${ }^{\mathrm{LO}}$ and NP-DNA- $40^{\mathrm{HI}}$, many nanoparticles were observed in the macrophages of the red pulp and in the capsule for both treated groups; again, none were observed in the white pulp (Supplementary Figure 7). 
(A)

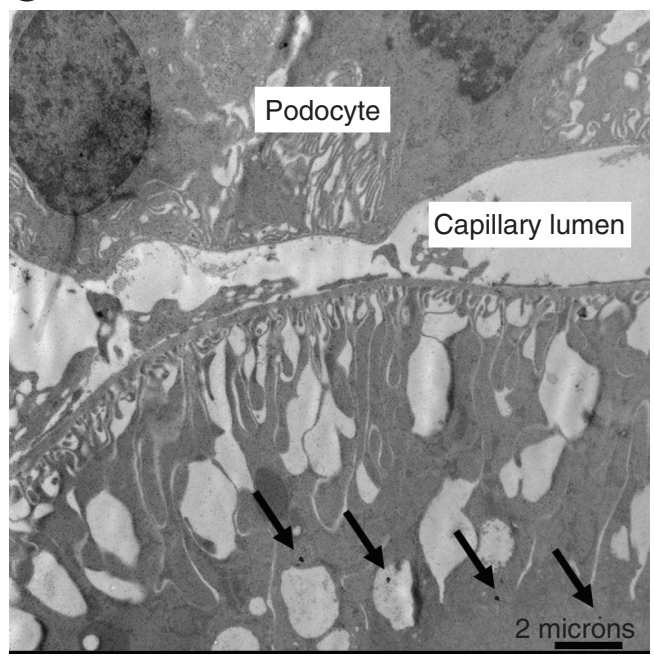

(B)

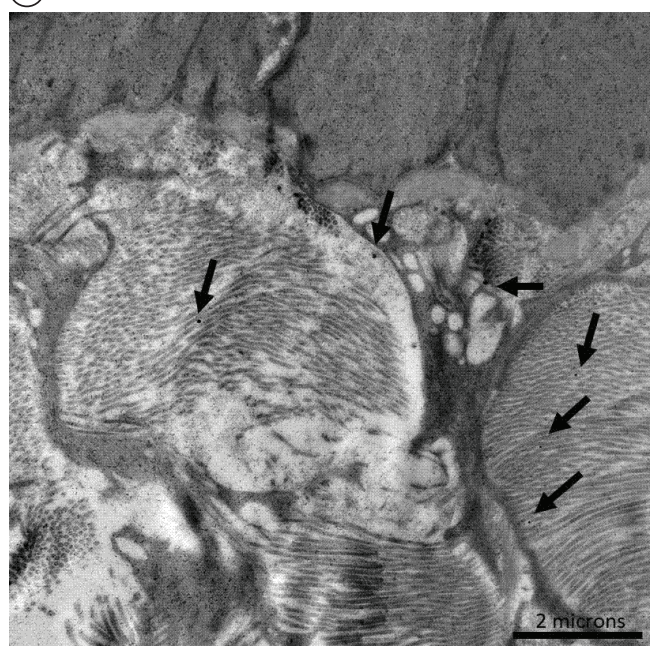

Figure 5. Transmission electron microscopy images from the cortex of the rat kidney following injection with glyconanoparticles. Nanoparticles arrowed are located in the Bowman's capsule (A) and in the brush border of the proximal tubular epithelium (B).

\section{Cellular distribution in the brain}

A small proportion of the injected nanocarriers accumulated in brain in this study, but there was no significant difference between the amounts in each treatment group (Figures $2 \& 3$ ). Previous studies have shown that glucosecoated GNPs move rapidly within the brain and can be found in neurons, astrocytes and endothelium within 10 min of infusion [12]. In this study, light microscopy of coronal sections of brain with silver enhancement showed darker staining in choroid plexus and median eminence that lack a full BBB. Some staining was also observed in striatum and areas of the cortex (Supplementary Figure 8). However, there was no consistent difference in staining of the ipsilateral (injected) and contralateral side of the coronal sections or of sections from rats that had received different treatments.

TEM images of areas of the cerebral cortex of animals injected with NP-DNA- $40^{\mathrm{LO}}$ showed silver-stained GNPs in endothelial cells, pericytes and astrocytes (Figure 7). A similar distribution of nanoparticles was found in animals in the other two treatment groups.

\section{Discussion}

The key finding from this study was that addition of a $40 \mathrm{nt}$ dsDNA cargo to the $2 \mathrm{~nm}$ gold glyconanoparticles caused a major change in their tissue distribution-accumulation in the kidney was almost completely blocked, and nanoparticles became primarily localized in liver and spleen (Figures $2 \& 3$ ). Note that the comparison in this study is between nanoparticles with a DNA cargo (NP-DNA-40) and nanoparticles without a cargo (NP-Gal, control). We did not include a saline only control because the two methods used, ICP-MS and silver-enhanced electron 
(A)

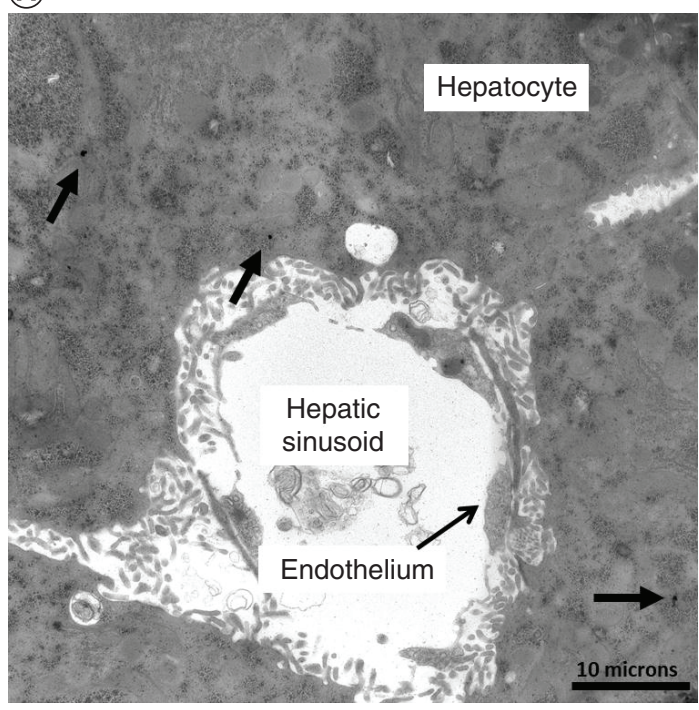

(B)

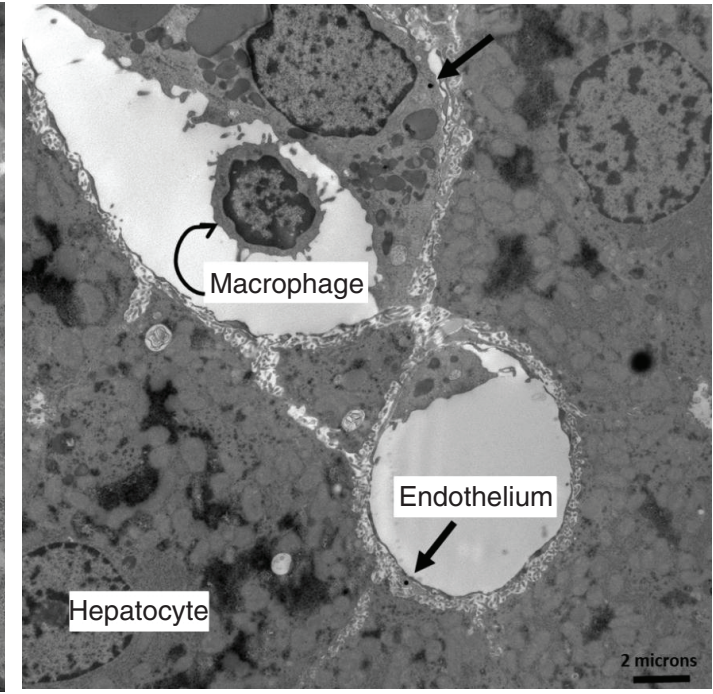

(C)

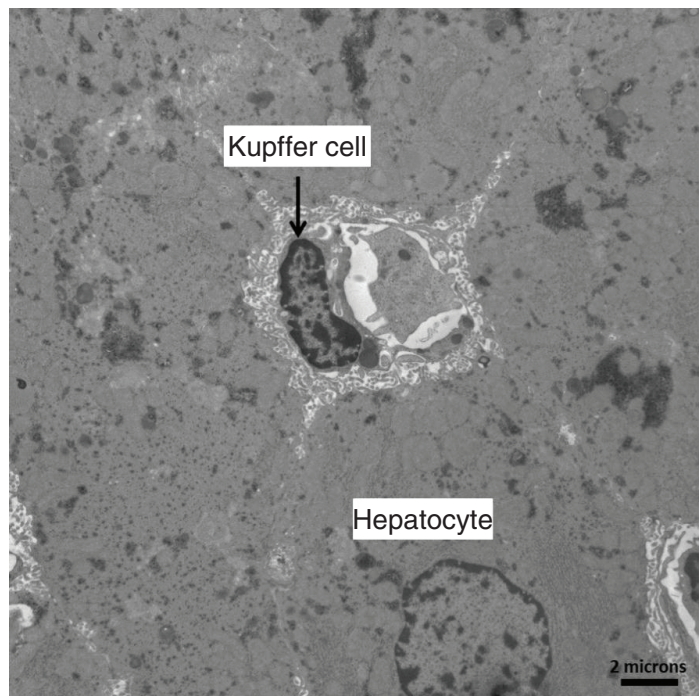

Figure 6. Representative transmission electron microscopy images of rat liver following injection with NP-DNA-40 ${ }^{\text {LO }}$ (A) and NP-DNA-40 ${ }^{\mathrm{HI}}$ (B \& C). Nanoparticles arrowed were primarily localized in the cytoplasm of hepatocytes with smaller numbers in the endothelium. They were not seen in macrophages or Kupffer cells.

microscopy, both detect gold, and we have previously noted that if no gold is injected, none can be detected in the tissues [12].

The design of this study was intended to determine the fate of the nanoparticles immediately after injection and to allow comparison with a previous study using similar nanoparticles [12]. It does not, however, provide information on the long-term persistence of the nanoparticles in different tissues. Previous work has implied that initial tissue distribution of gold NPS is strongly related to their surface coating, whereas persistence is related to size - smaller NPs are cleared relatively quickly [18]. Even within the 10-min timeframe of the experiments described here, the nanoparticles were almost completely cleared from the blood. In this respect, our findings are similar to those seen with 22-nm dextran-coated GNPs (no DNA cargo) injected into mice, where $90 \%$ of the injected dose was cleared from blood within $20 \mathrm{~min}$, and nanoparticles were subsequently found (at $24 \mathrm{~h}$ ), primarily in liver and spleen [19]. However, in contrast to that study, the smaller GNPs used here were localized in the cytosol of hepatocytes, with very few in phagocytic cells. This difference in distribution may be related to the nanoparticle size/charge, the presence of the DNA cargo or the time at which the organs were sampled. For example, at $\mathrm{pH} 7.5$ the addition of 
(A)

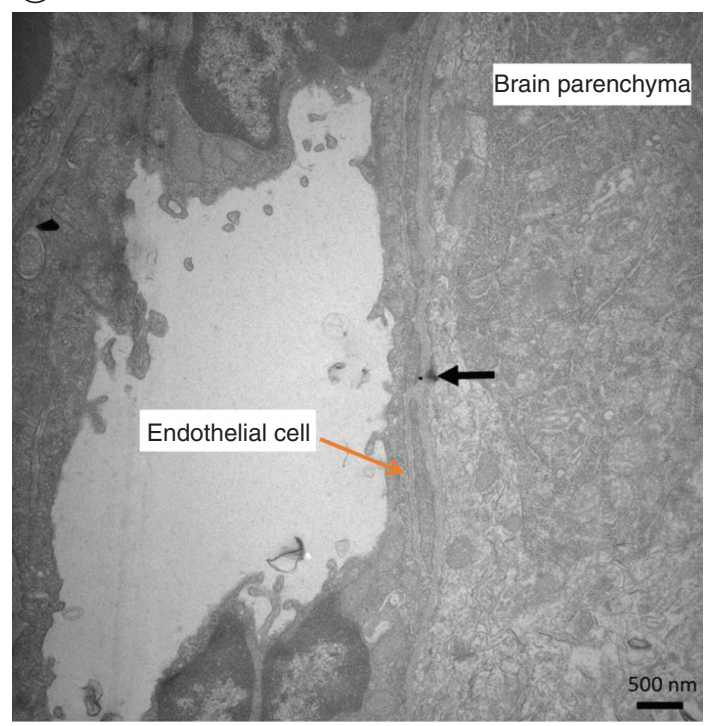

(B)

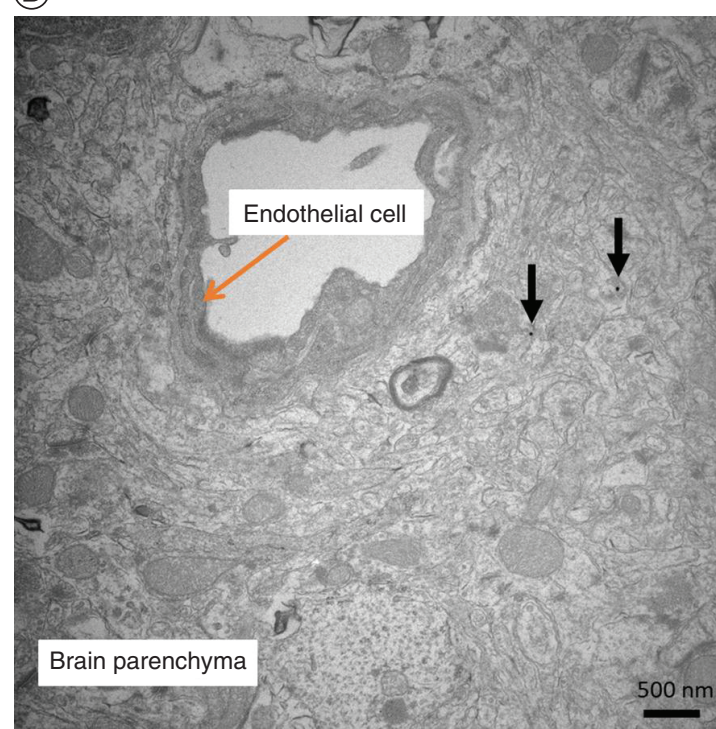

(c)

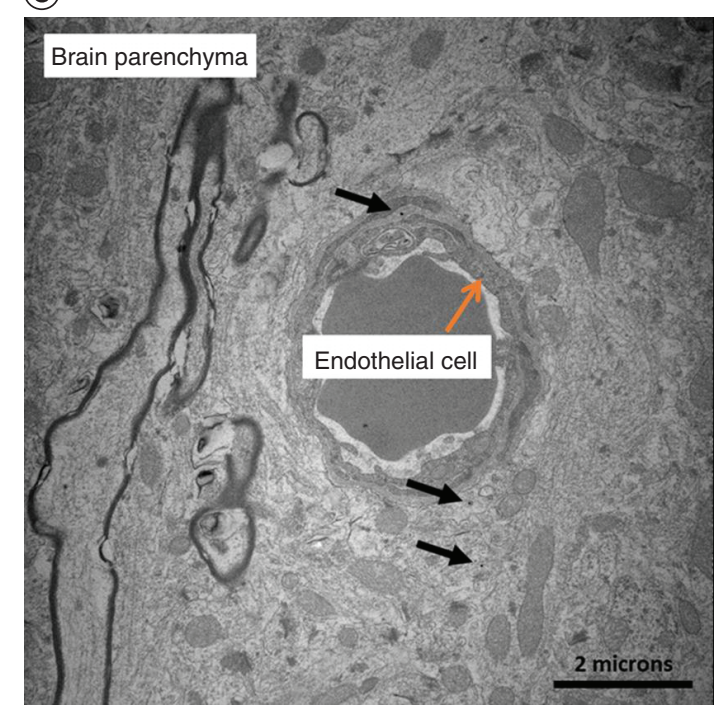

Figure 7. Transmission electron microscopy images of the cerebral cortex of a rat injected with NP-DNA-40 20.

Nanoparticles (arrows) were detected in brain endothelium (A), astrocytes in brain parenchyma (B) and in a pericyte and astrocytes (C).

the DNA oligonucleotides (pKa 2) changes the surface charge from near-neutral to strongly negative and increased the hydrodynamic diameter of the nanoparticles from 1.8 up to $7 \mathrm{~nm}$ (Supplementary Figure 1).

The low levels of oligonucleotide-coated GNPs in kidney in our study may be because NP-DNA- $40^{\mathrm{LO}}$ and NP-DNA- $40^{\mathrm{HI}}$ are larger in size than the unconjugated NP-Gal. Within the kidney, the NP-Gal base nanoparticles were localized throughout the organ, including the renal pelvis. In contrast, the NP-DNA- $40^{\mathrm{HI}}$ nanoparticles were confined to the Bowman capsule/glomerulus, whereas staining with the NP-DNA- $40^{\mathrm{LO}}$ formulation was more widely distributed in the cortex (Figure 4). The simplest explanation for these findings is that the progressive increase in size of the NPs with bound DNA, limits their ability to cross the glomerular filtration barrier. The attachment of additional oligonucleotides increases the size of the nanoparticles from 3 to $7 \mathrm{~nm}$, making them too large to cross the fenestrated glomerular endothelium, which has a cut-off of approximately $5 \mathrm{~nm}$ [11]. Hence, the smaller Gal-NP can move through the nephron and are seen in the brush border of the tubular epithelium and the renal pelvis. 
Endothelium has a strong negative charge due the glycocalyx, which is substantially lacking in vitro [20,21]. Therefore, an alternative possibility is that the negative charge of the DNA inhibits NP-DNA access to the endothelial surface in the glomerulus. However, in vivo it is not certain that these nanoparticles would be negatively charged. In the presence of serum, charged GNPs bind serum proteins of the opposite charge, which reduces or even reverses their charge [22,23]. For these reasons, the most likely explanation for the failure of NP-DNA to enter the nephron is that they are larger than the basic NP-Gal carrier.

Many studies have noted that nanoparticles $>10 \mathrm{~nm}$ accumulate in the liver [22,24] due to uptake by Kupffer cells and mononuclear phagocytes. However, we observed that the NPs were only in hepatocytes. We considered the possibility that the nanoparticles were binding to a 'galactose receptor'. Rat hepatocytes and liver macrophages have two different receptors that bind glycoproteins, which are both inhibited by $N$-acetylgalactosamine [25]. It seems unlikely that the enhanced uptake of NP-DNA- 40 by the hepatocytes can be explained by this galactose receptor; the receptor recognizes galactosyl groups on glycoproteins linked by their $\mathrm{C} 1$ atom, whereas the galactose attached to the GNPs is linked at C6, and therefore presented in a different orientation to that recognized by the receptor. Furthermore, the NP-Gal nanoparticles are not taken up in significant amounts in the liver (Figures $2 \& 3$ ). This implies that it is the addition of the DNA that alters the ability of hepatocytes to take up the nanoparticles. In this case the change in tissue distribution is less likely to be due to the increase in size of the nanocarriers but is instead due to the high negative charge imparted by the DNA cargo.

Although the addition of DNA reduced losses of the nanoparticles through the kidney, the level of uptake by the brain, lung or heart was not significantly increased. One aim of the research program has been to develop a nanocarrier that can cross the BBB in vivo. Intracarotid injection was originally chosen as a method of delivery, that might favor uptake of the nanocarriers by brain endothelium; previous studies had shown that gold glyconanoparticles do enter the brain preferentially on the injected side [12]. In this study, there was no significant difference in the brain uptake when intracarotid and intravenous infusion was compared. This finding suggests that during the first pass through the cerebral circulation, only a small proportion of the nanocarriers pass the BBB, and hence intracarotid injection only offers a small advantage over i.v. injection. However, once in the brain, nanoparticles with bound DNA were observed in glial cells, up to several microns from the nearest blood vessel (Figure 7). They had therefore moved rapidly within the brain, at a similar speed to unmodified gold glyconanoparticles [12].

While the addition of DNA to these nanocarriers has solved the problem of losses through kidney, it has not resolved how to increase transport across barrier-type brain endothelium. To increase passage of these NP-DNA across the BBB in vivo, it would be necessary to target them to brain endothelium [26]. For this purpose, the transferrin and low-density lipoprotein (LDL) receptors are selectively expressed on brain endothelium [27] and could be suitable targets for promoting vesicular transcytosis of nanoparticles up to $100 \mathrm{~nm}$. For example, larger pegylated GNPs $(25-32 \mathrm{~nm}$ ) have been treated with antibodies to the transferrin receptor to increase their uptake by brain endothelium [28]. This approach is less suitable for the glyconanoparticles used here because a targeting antibody $(6.4 \mathrm{~nm})$ is larger than the nanocarrier and cargo DNA.

An alternative approach is the use of targeting peptides. Synthetic polypeptides that bind to the transferrin receptor or LDL receptor have been used to target therapeutic agents to the brain $[29,30]$. These peptides can be modified by addition of a terminal cysteine residue, allowing them to be attached to the GNPs by an exchange reaction. For this reason, our current work on this project is aimed at increasing uptake of the nanocarriers by brain endothelium, using targeting peptides of $1-2 \mathrm{kDa}$, which do not add significantly to the size of the nanocarrier.

Although the unmodified NP-DNA do not enter the brain in large amounts, they do have great potential for gene therapy directed to liver hepatocytes. On the basis of the size of the animals used in the study $(\sim 300 \mathrm{~g})$, and an estimate of average liver weight of $12 \mathrm{~g}$, the amount of gold in the liver of rats treated with NP-DNA was $2 \mu \mathrm{g} / \mathrm{g}$ (Figure 2) or $24 \mu \mathrm{g}$. Because these animals were injected with $200 \mu \mathrm{g} / \mathrm{kg}$ of gold, the amount in the liver $(24 \mu \mathrm{g})$ represents $40 \%$ of the total injected dose $(60 \mu \mathrm{g})$. This contrasts with the $0.4 \%$ entering the brain by similar calculation. Moreover, this high level of uptake into the liver occurred within $10 \mathrm{~min}$ of infusion. Oligonucleotides of 40-nt length were specifically selected in this study as a suitable size for gene therapy. Because the GNPs rapidly and efficiently enter the cytoplasm of hepatocytes, they are very well suited for delivering gene therapy for liver diseases [31].

\section{Conclusion}

This study has shown that it is essential to consider how the characteristics of a cargo molecule, in this case dsDNA oligonucleotides, affects the biodistribution of a nanocarrier. This is particularly true where the cargo molecule is 
attached to the outside of the carrier because it can then directly interact with tissue cells and endothelial cells, which have distinctive tissue-specific properties. Addition of cargo molecules to nanoparticles $<5$ nm significantly increases their size and reduces their ability to pass glomerular endothelium. The charge of the cargo also affects ability to interact with cells in tissues including hepatocytes and mononuclear phagocytes.

\section{Summary points}

- Gold glyconanoparticles (2 nm) with a covalently attached shell of C2 galactose were used as nanocarriers for a DNA cargo in vivo.

- The dsDNA oligonucleotides (40 nt) were covalently attached to the nanocarriers by an exchange reaction, producing 1-6 DNA molecules per nanocarrier.

- Formulations with low levels of bound DNA and high levels of bound DNA were compared with nanoparticles with no DNA cargo.

- The nanocarriers were infused into rats either intravenously or into the carotid artery and allowed to circulate for $10 \mathrm{~min}$ before organs were perfused and harvested.

- The tissue distribution of the nanocarriers was determined by inductively coupled plasma mass spectrometry (ICP-MS) and transmission electron microscopy.

- Addition of DNA to the carriers completely blocks their localization in kidney but promotes uptake by liver hepatocytes and splenic macrophages.

- The amounts localizing in the heart, lung and brain were not affected by addition of the DNA.

- The study demonstrates that cargo molecules such as DNA on small nanocarriers can substantially increase their size, alter their charge and radically affect tissue distribution.

Supplementary data

To view the supplementary data that accompany this paper please visit the journal website at: www.futuremedicine.com/doi/suppl/10.2217/nnm-2020-0469

Financial \& competing interests disclosure

Financial support was received from the Sheffield Hospitals Trust. The authors have no other relevant affiliations or financial involvement with any organization or entity with a financial interest in or financial conflict with the subject matter or materials discussed in the manuscript apart from those disclosed.

No writing assistance was utilized in the production of this manuscript.

\section{Ethical conduct of research}

The authors state that they have obtained appropriate institutional review board approval or have followed the principles outlined in the Declaration of Helsinki for all human or animal experimental investigations.

\section{Open Access}

This work is licensed under the Creative Commons Attribution 4.0 License. To view a copy of this license, visit http://creativecomm ons.org/licenses/by/4.0/

\section{References}

Papers of special note have been highlighted as: $\bullet$ of interest

1. Deverman BE, Ravina BM, Bankiewicz KS et al. Gene therapy for neurological disorders: progress and prospects. Nat. Rev. Drug Discov. 17(10), 641-659 (2018).

2. Crawford L, Rosch J, Putnam D. Concepts, technologies, and practices for drug delivery past the blood-brain barrier to the central nervous system. J. Control. Release 240, 251-266 (2016).

3. Juliano RL. The delivery of therapeutic oligonucleotides. Nucleic Acids Res. 44(14), 6518-6548 (2016).

- Discusses how to overcome tissue barriers to the delivery of oligonucleotides for siRNA and miRNA-based therapies. This is related to how oligonucleotides can be chemically modified to maintain stability in the plasma and how the carriers can evade the mononuclear phagocyte system.

4. Gromnicova R, Davies H, Sreekanthreddy P et al. Glucose-coated gold nanoparticles transfer across human brain endothelium and enter astrocytes in vitro. PLoS ONE 8(12), e81043 (2013).

5. Gromnicova R, Kaya M, Romero IA et al. Transport of gold nanoparticles by vascular endothelium from different human tissues. PLoS ONE 11(8), e0161610 (2016).

6. Verma A, Uzun O, Hu Y et al. Surface-structure-regulated cell-membrane penetration by monolayer-protected nanoparticles. Nat. Mater. 7(7), 588-595 (2008). 
7. Male D, Gromnicova R, McQuaid C. Gold nanoparticles for imaging and drug transport to the CNS. Int. Rev. Neurobiol. 130, 155-198 (2016).

8. Ding Y, Jiang Z, Saha K et al. Gold nanoparticles for nucleic acid delivery. Mol. Ther. 22(6), 1075-1083 (2014).

9. Vales G, Suhonen S, Siivola KM et al. Genotoxicity and cytotoxicity of gold nanoparticles in vitro: role of surface functionalization and particle size. Nanomaterials (Basel) 10(2), 271 (2020).

10. Midatech Pharma PLC. Midatech's MTX110 receives orphan designation for DIPG. Exhibit 99.1 (2019). www.sec.gov/Archives/edgar/data/1643918/000121465919006612/ex99_1.htm

11. Haraldsson B, Nystrom J, Deen WM. Properties of the glomerular barrier and mechanisms of proteinuria. Physiol. Rev. 88(2), 451-487 (2008).

12. Gromnicova R, Yilmaz CU, Orhan $\mathrm{N}$ et al. Localization and mobility of glucose-coated gold nanoparticles within the brain. Nanomedicine (Lond). 11(6), 617-625 (2016).

13. Fatima N, Gromnicova R, Loughlin J et al. Gold nanocarriers for transport of oligonucleotides across brain endothelial cells. PLoS ONE 15(9), e0236611 (2020).

- Describes the transport of gold nanocarriers across a 3D model of the human blood-brain barrier in vitro. Addition of DNA cargo to the nanocarriers enhances transcytosis of the DNA across the endothelium up to 50-fold.

14. Brust M, Walker M, Bethell D et al. Synthesis of thiol-derivatised gold nanoparticles in a two-phase liquid-liquid system. Chem. Commun. (Camb) 7, 801-802 (1994).

15. Taton TA. Preparation of gold nanoparticle-DNA conjugates. Curr. Protoc. Nucleic Acid Chem. 9(1), 12.2.1-12.2.12 (2002).

16. Skotland T, Iversen TG, Sandvig K. New metal-based nanoparticles for intravenous use: requirements for clinical success with focus on medical imaging. Nanomedicine 6(6), 730-737 (2010).

17. Papasani MR, Wang G, Hill RA. Gold nanoparticles: the importance of physiological principles to devise strategies for targeted drug delivery. Nanomedicine 8(6), 804-814 (2012).

18. Khlebtsov N, Dykman L. Biodistribution and toxicity of engineered gold nanoparticles: a review of in vitro and in vivo studies. Chem. Soc. Rev. 40(3), 1647-1671 (2011).

19. Bailly A-L, Corread F, Popov A et al. In vivo evaluation of safety, biodistribution and pharmacokinetics of laser-synthesized gold nanoparticles. Sci. Rep. 9, 12890 (2019).

20. Weinbaum S, Tarbell JM, Damiano ER. The structure and function of the endothelial glycocalyx layer. Annu. Rev. Biomed. Eng. 9 , 121-167 (2007).

21. Potter DR, Damiano ER. The hydrodynamically relevant endothelial cell glycocalyx observed in vivo is absent in vitro. Circ. Res. 102(7), 770-776 (2008).

22. Dobrovolskaia MA, Patri AK, Zheng J et al. Interaction of colloidal gold nanoparticles with human blood: effects on particle size and analysis of plasma protein binding profiles. Nanomedicine 5(2), 106-117 (2009).

23. Alkilany AM, Murphy CJ. Toxicity and cellular uptake of gold nanoparticles: what we have learned so far? J. Nanoparticle Res. 12(7), 2313-2333 (2010).

- Considers not only the effects of size and shape on the cellular interactions of gold nanoparticles but also how they interact with plasma proteins and the formation of a protein corona, which critically affects uptake by different cell types.

24. Longmire M, Choyke PI, Kobayashi H. Clearance properties of nano-sized particles and molecules as imaging agents: considerations and caveats. Nanomedicine (Lond). 3(5), 703-717 (2008).

25. Kempka G, Kolb-Bachofen V. Galactose-specific receptors on liver cells. I. Hepatocyte and liver macrophage receptors differ in their membrane anchorage. Biochim. Biophys. Acta 847(1), 108-114 (1985).

26. Lajoie JM, Shusta EV. Targeting receptor-mediated transport for delivery of biologics across the blood-brain barrier. Annu. Rev. Pharmacol. 55, 613-631 (2015).

27. Sharma G, Sharma AR, Sang-Soo L et al. Advances in nanocarriers enabled brain targeted drug delivery across blood brain barrier. Int. J. Pharm. 559, 360-372 (2019).

- Summarizes progress in the development of nanocarriers for CNS drug delivery, and discusses the considerable problems that remain in promoting transport of larger therapeutic biomolecules.

28. Amani H, Mostavavi E, Alebouyeh MR et al. Would colloidal gold nanocarriers present an effective diagnosis of treatment for ischaemic stroke? Int. J. Nanomed. 14, 8013-8031 (2019).

29. Demeule M, Regina A, Che C et al. Identification and design of peptides as a new drug delivery system for the brain. J. Pharmacol. Exp. Ther. 324(3), 1064-1072 (2008).

30. Huang R, Ma H, Guo Y et al. Angiopep-conjugated nanoparticles for targeted long-term gene therapy of Parkinson's disease. Pharm. Res. 30(10), 2549-2559 (2013).

31. Baruteau J, Waddington SN, Alexander IE, Gissen P. Gene therapy for monogenic liver diseases: clinical successes, current challenges and future prospects. J. Inherit. Metab. Dis. 40(4), 497-517 (2017). 